\title{
Research Article \\ Optical Connecting of Fibers by Laser Beams Propagating from the Fibers Edges
}

\author{
Sergey Nikolayevich Mensov ${ }^{1,2}$ and Yuri Victorovich Polushtaytsev² \\ ${ }^{1}$ Nizhny Novgorod State University, 23 Gagrin Avenue, Nizhny Novgorod 603950, Russia \\ ${ }^{2}$ Institute of Organometallic Chemistry of RAS, 49 Tropinin Street, Nizhny Novgorod 603950, Russia
}

Correspondence should be addressed to Sergey Nikolayevich Mensov, mensov@rf.unn.ru

Received 28 June 2007; Accepted 14 January 2008

Recommended by Mustafa Abushagur

A possibility to connect nonprecise positioned fibers in photopolymerizable compositions is under discussion in this paper. The processes of optical synthesis of connective waveguiding structures forming in such mediums directly by the radiation leaving the edges of connecting fibers are investigated numerically and experimentally as well. It was shown that nonlinear interaction of the light beams allows to connect misaligned and transversally shifted fibers with high efficiency.

Copyright ( 2008 S. N. Mensov and Y. V. Polushtaytsev. This is an open access article distributed under the Creative Commons Attribution License, which permits unrestricted use, distribution, and reproduction in any medium, provided the original work is properly cited.

\section{INTRODUCTION}

At fiber optical networking, a significant attention is focused on fibers interconnecting. Traditional methods of fibers joining are quite precise: optical losses in connective units considerably depend on accuracy of the fiber edges mutual positioning [1-13]. It became possible to create waveguiding channels in modern transparent photopolymerizable compositions (PPC) by light directly [1-6]. Nonlinear processes of optical radiation interaction with such substances can be used to synthesize connective elements for fiber optics [7-12]. Crossing light beams are capable to exert mutual influence in the PPC [8-10]. Due to essential nonlinearity of the polymerization process, the propagation trajectory of the interacting beams after the crossing and, hence, the shape of formed channels can differ from straight tracks radically. Moreover, bending of the forming channels is possible for transversally shifted (initially nonintersecting) parallel light beams owing to significant diffractive divergence of the radiation leaving the edges of modern optical fibers [11, 12]. In both cases, this stipulates that creation of a single waveguiding structure directing radiation to the counter channel is possible.

In this paper, we considered the possibility of optical connecting of misaligned and transversally shifted fibers in the photopolymerizable compositions and investigated the processes of optical synthesis of connective waveguiding structures forming in such mediums directly by the radiation leaving the edges of connecting fibers.

\section{OPTICAL JOINING OF TRANSVERSALLY SHIFTED FIBERS}

Considerable diffractive divergence of the radiation propagating from a fiber causes great losses in the connective unit if the edges of connected waveguides are spaced even on a small gap [13]. On the contrary, to provide edges with a clearance becomes a necessary condition to create their effective optical joining in the PPC even if the fibers are transversally shifted (see Figure 1).

The analysis of optical joining of two fibers was carried out in the frameworks of the following model. The segments of graded-index fibers with the Gaussian shape of the refraction parameter (Figure 1) by the width of $2 a$ were considered as the optical elements to be connected. The axes of the fibers were transversally displaced on the distance $2 \rho$. Nonlinear interaction of the radiation with the initially homogeneous PPC was considered in the gap $2 l_{f}$ between the fiber edges. The following system of wave and material equations was used at the numerical simulation [12-15]: 


$$
\begin{aligned}
& \pm 2 i k \cdot \frac{\partial E_{1,2}^{\prime}(x, z, H)}{\partial z} \\
& =\frac{\partial^{2} E_{1,2}^{\prime}(x, z, H)}{\partial x^{2}}+2 k^{2} \cdot \delta n_{\max } \\
& \cdot \Delta n^{\prime}(x, z, H) \cdot E_{1,2}^{\prime}(x, z, H), \\
& \Delta n^{\prime}(x, z, H) \\
& = \begin{cases}1-\exp \left\{-H(x, z, t)^{\gamma}\right\}, & |z| \leq l_{\mathrm{f}}, \\
\exp \left\{-\frac{x^{2}}{a^{2}}\right\}, & l_{\mathrm{f}}<|z| \leq l_{\mathrm{f}}+l_{\mathrm{w}},\end{cases} \\
& H(x, z, t)=\frac{E_{0}^{2}}{H_{0}} \int_{0}^{t}\left[J_{1}^{\prime}(x, z, \tau)+J_{2}^{\prime}(x, z, \tau)\right] d \tau,
\end{aligned}
$$

with the stationary boundary conditions:

$$
E_{1,2}(x, \pm L, H)=E_{0} \cdot \exp \left\{-\frac{(x \mp \rho)^{2}}{2 a^{2}}\right\}
$$

where $E^{\prime}=E / E_{0}, J^{\prime}(x, z, H)=\left|E^{\prime}(x, z, H)\right|^{2}, \Delta n^{\prime}=\Delta n /$ $\Delta n_{\max }, \delta n_{\max }=\Delta n_{\max } / n_{0}, n_{0}$-monomer refractive index, $\Delta n_{\max }$ - maximal change on the PPC refractive index, $H$ normalized exposure, $H_{0}$ and $\gamma$-contrast parameters of the composition, $a$-half-width of the beam, and $k$ - wave number. In the left-hand part of the wave equation in the system (1), the sign "plus" corresponds to direct propagation of the radiation, and the sign "minus" to contrary direction. This equation was solved independently for each of the beams. Modification of the medium was assumed in accordance with the summary intensity distribution. Both, evolution of the refraction parameter $\Delta n^{\prime}(x, z, H)$ in the forming polymeric channel and the behavior of the radiation in it were under investigation at the numerical simulation. Fibers with the value of guiding parameter $G=k a \cdot\left(2 \Delta n_{\max } / n_{0}\right)^{0.5} \approx 4.2$ were under consideration $\left(\delta n_{\max }=0.01, \gamma=3, a=3 \mu \mathrm{m}\right.$, $2 \pi / k=0.63 \mu \mathrm{m})$. Their length $l_{w} \sim 14 k a^{2}$ provided formation of inherent mode structures of the radiation on the edges of the connecting fibers.

In accordance with Figure 2, at the initial stages of the self-channeling process [3], each waveguiding channel forms with almost straight trajectory. Mutual influence increases in the convergence of the "leading edges" of the polymeric channels forming by light. If the transversal distance between the channels (at $z=0$ ) becomes less than a beam width, a single waveguiding channel forms (Figure 2(a)). In this case, each of the beams propagates through the channel, already formed by the opposite beam.

Effectiveness of the beams interaction decreases with the increment of transversal shifting $2 \rho$, and so residual transversal distance between the channels $(z=0)$ will exceed $2 a$. In this case, each of the channels continues growth behind the plane $z=0$. The interaction will not stop inasmuch as thin light beams possess considerable diffractive divergence and their intensity cannot be completely localized in gradient fibers. Accordingly, behind the plane $z=0$, mutual inclination increment is caused by the influence of opposite beam propagating through the formed segment of the gradient channel. Finally, trajectory of the beam $E_{1}$ crosses the

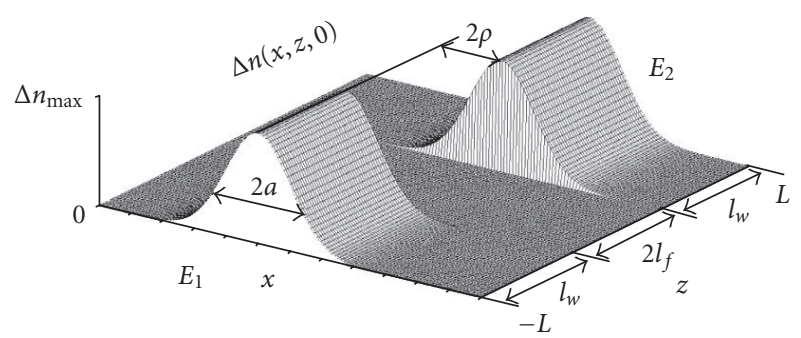

FIGURE 1: Mutual location of connected fibers: $l_{w}$ is the fiber length; $2 l_{f}$ is the distance between the end-faces of fibers (gap); and $2 \rho$ is the transversal displacement of fibers.

trajectory of the beam $E_{2}$ and a loop-shaped structure forms (Figure 2(b)).

Domain of initial parameters $\rho$ and $l_{f}$ is divided by the curve into two areas (Figure 3 ) according to the residual transversal distance between the beams when the "leading edges" of synthesized channels reach the plane $z=0$. The more initial shifting of the beams $2 \rho$, the grater should be clearance $2 l_{f}$ between entrance planes to form a single channel.

This dependence function conforms to the results of the linearized model for the process of bended waveguiding channels formation by the Gaussian beams of light $[11,12]$. According to this model, the effective mutual influence of counterpropagating beams occurs in the strongly determined domain of parameters $\left(\rho, l_{f}\right)$, when the direction of the propagation changes considerably. Therefore, to believe that for every value $\rho$ the clearance $2 l_{f}$ can be adjusted to provide a single channel formation is incorrect. The restriction is due to the fact that the parameters domain of the most effective mutual influence is located nearby $z=0$, where the maximal bending of the waveguiding channels is observed; and its typical longitudinal size is approximately $2 k a^{2}$ for different parameters of the beams. Outside this domain, formation of the channels occurs with almost straight trajectories.

Hence, optical connecting of fibers allows joining transversally shifted waveguides owing to possibility to form a single waveguiding channel in the PPC. According to the results of numerical analysis of the radiation propagation through the synthesized polymeric structure, the value of power losses does not exceed some percents and insignificantly depends on increment of the polymeric frame length (Figure 4(a)). For comparison, this value for the uniform gap between the fibers edges significantly depends both on the distance $2 l_{f}$ and on the shifting $2 \rho$ (Figure 4(b).

\section{CONNECTING OF NONPARALLEL FIBERS}

It is possible to create a single waveguiding channel even by the radiation propagating from the edges of the fibers with intersecting optical axes. The analysis for such mutual influence of two counterpropagating Gaussian light beams was based on numerical solving of the same system (1). The case, when $\rho=0$ and the optical axes of the fibers were inclined 

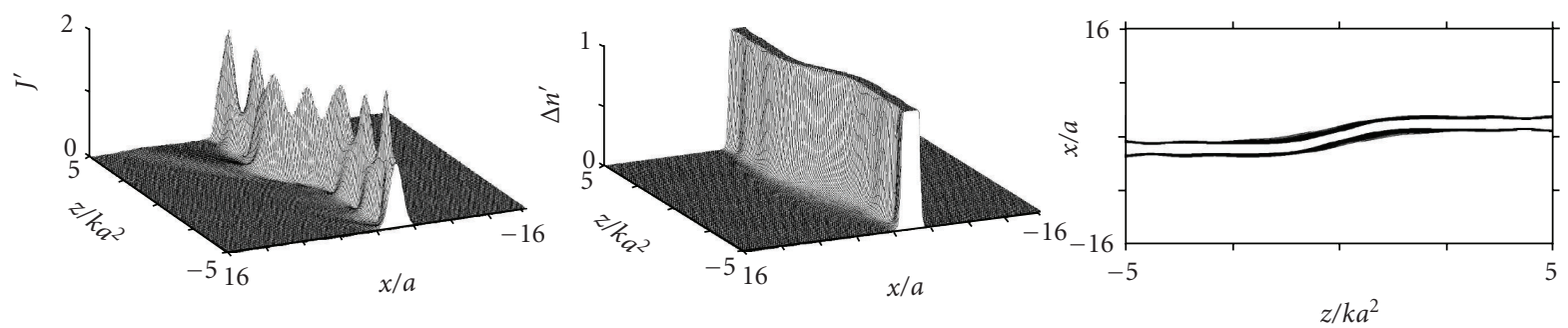

(a)
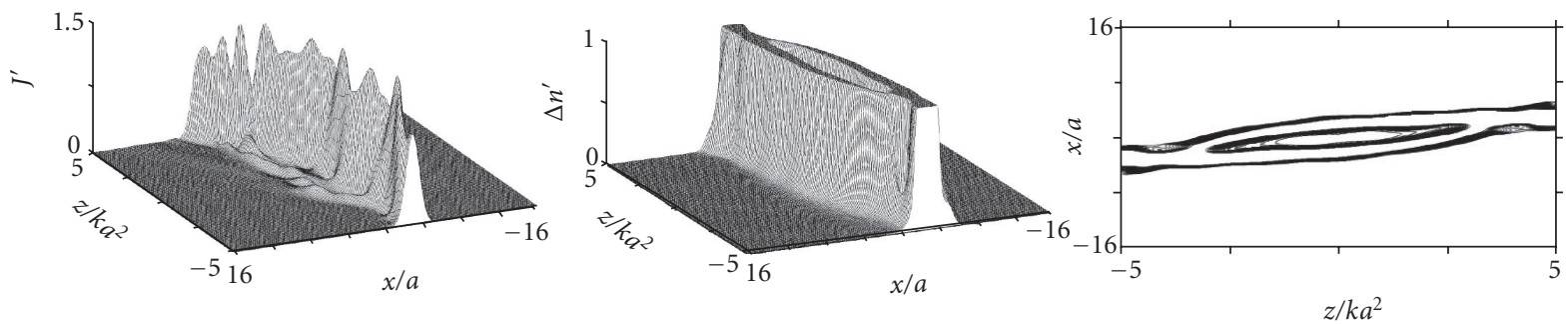

(b)

FIGURE 2: Numerical simulation results for the interaction process of nonintersecting light beams in the PPC $\left(l_{f}=10 \mathrm{ka} \mathrm{a}^{2}\right)$ : intensity distribution of the beam, propagating through the formed polymeric connector (a) and through the loop-shaped structure (b).

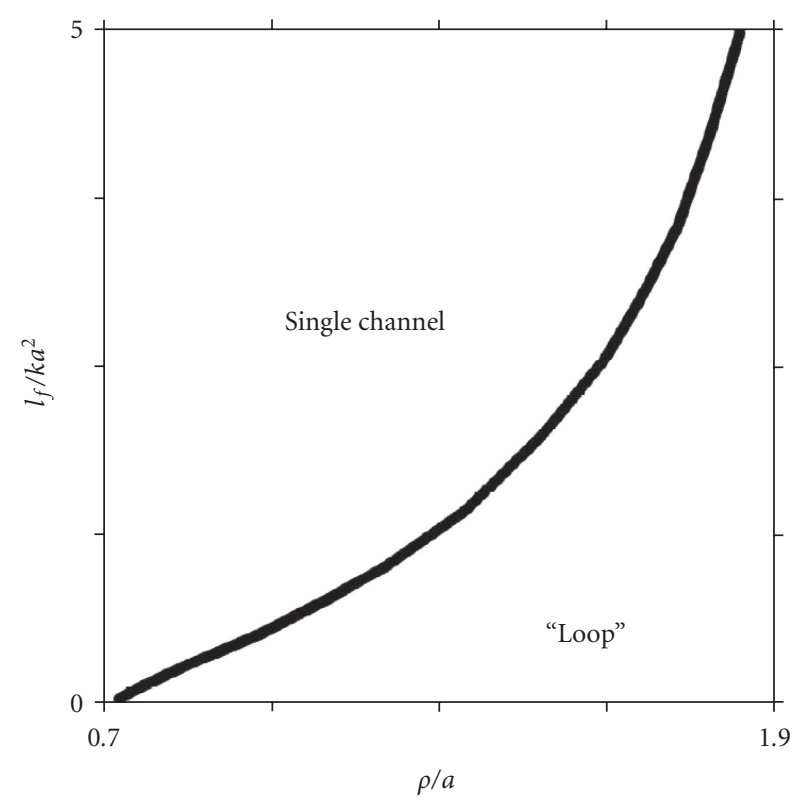

FIGURE 3: Condition of a single waveguiding channel formation in the PPC.

on the angle $\alpha$ to the axis $z$, was considered. The following boundary conditions were used:

$$
E_{1,2}(x, \pm L, H)=E_{0} \cdot \exp \left(-\frac{x^{2}}{2 a^{2}} \mp i k x \cdot \alpha\right) .
$$

There are two choices of a polymeric structure formation in the area of crossing of the beams leaving the fibers edges (Figure 5). For great values $\alpha$, when an independent selfchanneling of each of the beams is observed, a cross-shaped structure forms (Figure 5(a)). After crossing, the beams

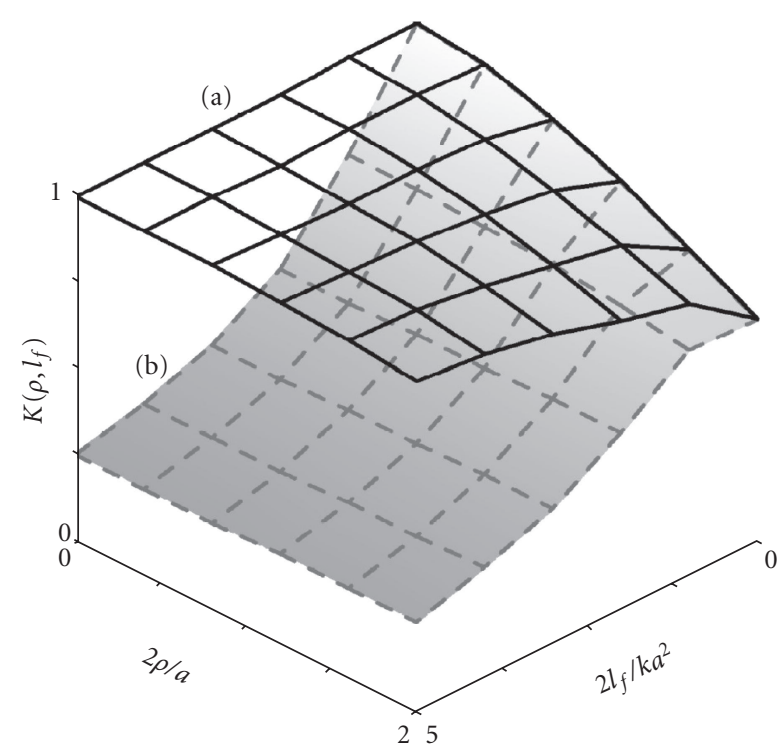

FIGURE 4: Dependence of energy transfer coefficient of connective unit on parameters $\rho$ and $l_{f}$.

propagate keeping initial direction and form straight channels. On the contrary, if the angle $\alpha$ is less than some critical value $\alpha_{\mathrm{cr}}$, an effect of the beams "mutual trapping" occurs-a single waveguiding structure is formed which turns the radiation to the opposite channel (Figure 5(b)). At that, the value $\alpha_{\text {cr }}$ nonproportionately increases, while the value of maximal relative additive to the PPC refraction index $\delta n_{\max }$ rises up (Figure 6).

This peculiarity of waveguiding structures formation conforms to the results of the linearized model for the interaction of intersecting Gaussian light beams [8, 9]. According 

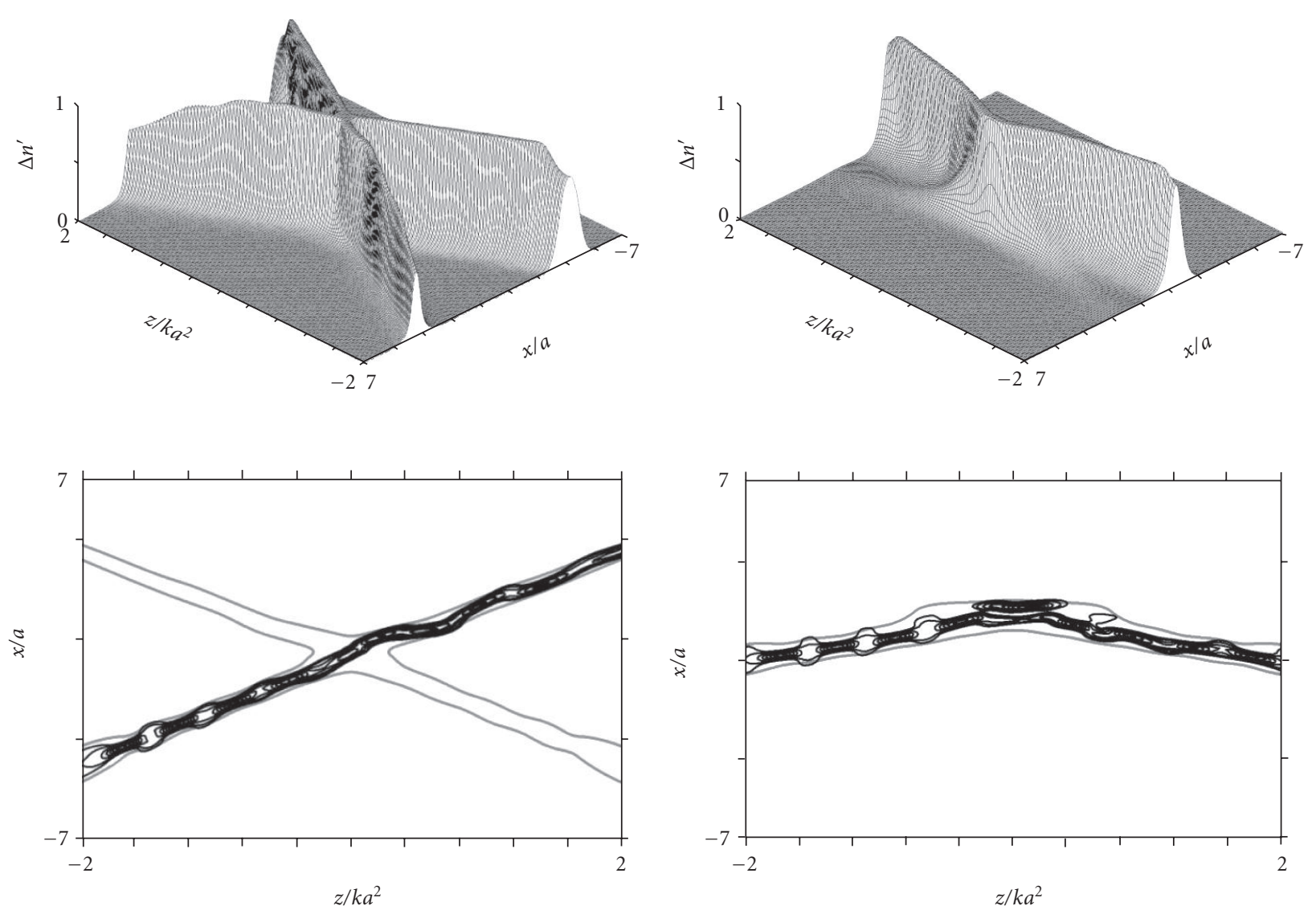

(a) crossing of the waveguiding channels, $\alpha=4^{\circ}$

(b) "mutual trapping" of the channels, $\alpha=1.3^{\circ}$

FIGURE 5: Numerical simulation results for interaction process of intersecting light beams in the PPC.

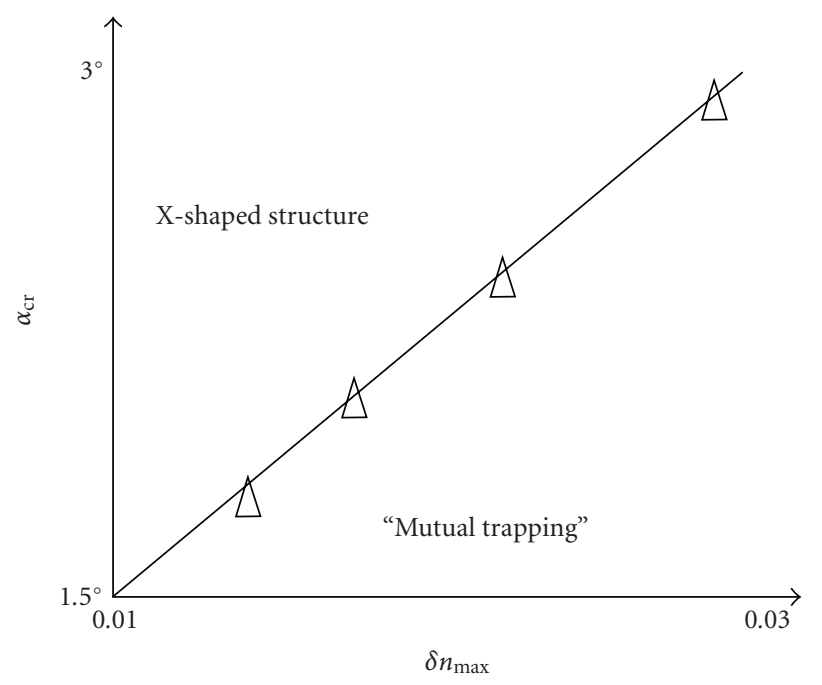

FIgURE 6: Dependence of the critical angle $\alpha_{\text {cr }}$ on the maximal relative change of the PPC refraction index $\delta n_{\max }$.

to this model, the condition of total internal reflection from a common polymeric frontier, forming in the PPC, determines the value $\alpha_{\mathrm{cr}}$. The frontier can be generated in the region of the beams intersection, despite the fact that even in initial distribution of the total intensity of the beams there are "forerunners" of cross-shaped polymeric structure formation. Generating of this reflecting inhomogeneity is caused by considerable nonlinearity of the PPC exposure characteristic, that is, difference in the polymerization velocities in the center of the region of the beams crossing and in the periphery.

\section{EXPERIMENTAL RESEARCH}

Experimental research of the fibers optical connecting process was carried out for photopolymerizable composition based on oligomer OCM-2 with quinone photoinitiator [15]. Its photosensitivity threshold is $0.65 \mu \mathrm{m}$. Low absorption of visible and infrared (IR) radiation is typical for this PPC and, correspondingly, nonlinear optical wave processes can be realized at great enough distances.

According to the scheme of the experiment (Figure 7), semiconductor laser modules 1 (wavelength is $0.63 \mu \mathrm{m}$ ) provided intensity in the fibers approximately $3 \mathrm{~mW} / \mathrm{mm}$. The laser beams were focused by short-focus lenses 2 at the edges of the fibers 3 , and their free edges were placed into the reactor with photopolymer 4 , which was made of two glass plates, transversally bounding the PPC layer. The layer was 


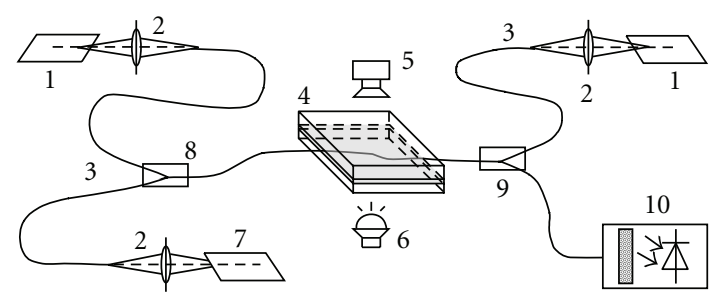

Figure 7: Optical scheme of experimental setting: 1-semiconductor lasers, 2-lenses, 3-fibers, 4-reactor with PPC, 5camcorder, 6-IR backlight, 7-IR laser, 8-summator, 9-splitter, and 10 — photodiode supported with IR filter.

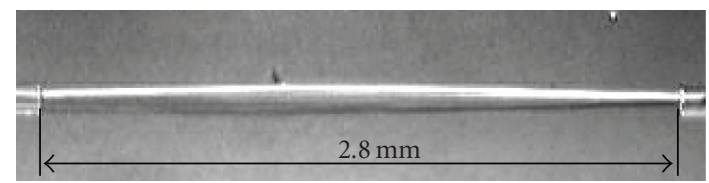

(a)

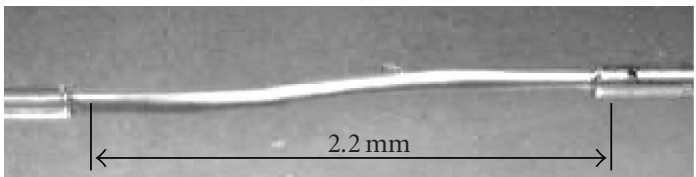

(b)

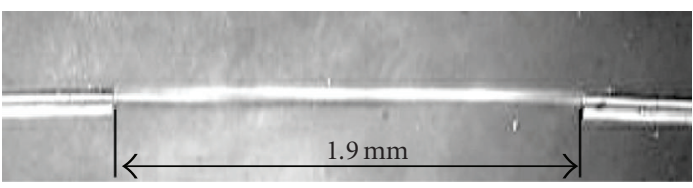

(c)

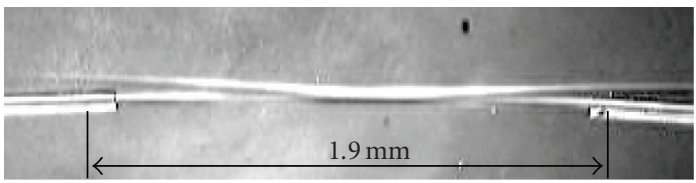

(d)

FIGURE 8: Experimentally synthesized polymeric structures formed under the radiation propagating from the edges of multimode fibers: at their coaxial positioning (a), at transversal shifting $2 \rho=$ $150 \mu \mathrm{m}(\mathrm{b})$, fibers are under inclination $2 \alpha=3^{\circ}$ (c), and $2 \alpha=6^{\circ}$ (d).

thick enough $(\sim 200 \mu \mathrm{m})$ to exclude probable influence of the reactor borders. Visualization of the optically formed polymeric structure was carried out with the camcorder 5 at lateral illumination by infrared light 6 , which does not cause any modification of the photopolymerizable medium. Mutual position of the fiber edges was adjusted by micromanipulators allowing changing transversal displacement and clearance between the edges. The scheme for measurement of energy transfer coefficient of synthesized connector contains IR laser 7 (wavelength $0.85 \mu \mathrm{m}$, intensity in the fiber $\sim 0.1 \mathrm{~W} / \mathrm{mm}^{2}$ ), summator 8 , splitter 9 and photodiode 10 supported with IR filter (KS17).

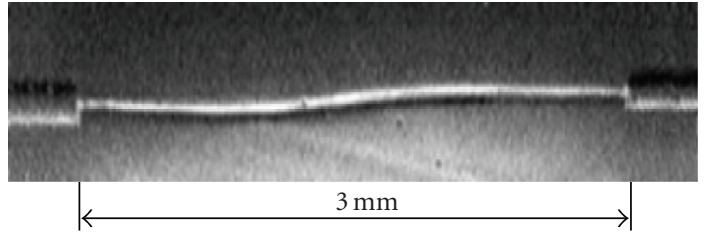

(a)

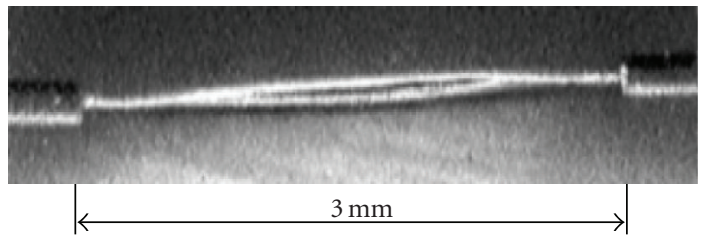

(b)

FIGURE 9: Experimentally synthesized polymeric structures formed under the radiation propagating from the edges of single-mode fibers: (a)—single channel $(2 \rho=20 \mu \mathrm{m})$, (b)—loop-shaped structure $(2 \rho=150 \mu \mathrm{m})$.

Experimental results of optical connecting of standard multimode fibers (the core is $50 \mu \mathrm{m}$ and diameter of the buffer is $125 \mu \mathrm{m}$ ) are shown in Figure 8 for different cases of their mutual locations. The value of the fiber guiding parameter respects to the parameter $G$ considered on the numerical simulation. The photographs of polymeric structures synthesized by the radiation leaving the fiber edges are shown in this figure. In the case of coaxial location of the fiber tips, the radiation forms a straight polymeric channel (Figure 8(a)). If the fibers are transversally shifted the trajectory of the beams, propagation is bended and nonrectilinear channel is formed as a result of mutual influence of the beams (Figure 8(b)). Mutual trapping is possible even if the fiber tips are inclined (Figure 8(c)); but crossing beams form a cross-shaped structure (Figure $8(\mathrm{~d})$ ) if the condition of mutual trapping is failed (Figure 6).

The results of theoretical calculations and computer simulation showed that the interaction route of several diffractive lengths $\left(\mathrm{ka}^{2}\right)$ is sufficient to create a single waveguiding channel. Nevertheless, a loop-shaped structure formation is possible on extended distances only: tens of diffractive lengths or tens of millimeters if multimode fibers are used. Therefore, research of loop-shaped structure formation was carried out for single-mode fibers (the core is $9 \mu \mathrm{m}$ and diameter of the buffer is $125 \mu \mathrm{m}$ ). Diffractive divergence of the radiation leaving the edge of fiber with thin core is high enough to provide effective interaction of the beams on the route of several millimeters (Figure 9 ).

The experimental setting (Figure 7) allowed to control quality of synthesized waveguiding structures. Energy transfer measurement showed that considered above polymeric connectors formed by light (Figures 8(a)-8(c) and 9) possess high transfer efficiency and they are capable to guide optical radiation to the opposite fiber with power losses not exceeding $5 \%$. 


\section{CONCLUSIONS}

So, nonlinear interaction of light beams leaving the edges of standard optical fibers allows connecting misaligned and transversally shifted fibers with high efficiency. A single waveguiding channel formation has been ascertained to be possible for both mutually tilted $\left(2 \alpha=3^{\circ}\right)$ and transversally shifted $(2 \rho=150 \mu \mathrm{m})$ fibers. Formed gradient polymeric structure keeps its shape after the exposure cessation. Owing to the PPC photosensitivity threshold (in the visible range) energy transfer coefficient of the connectors does not change even if comparatively energetic infrared radiation propagates through it. This allows using these polymeric connectors at the fiber optical networking directly.

\section{ACKNOWLEDGMENT}

This work was supported by Grants of RFBR no. 05-0332706-a and no. 06-03-08186-ofi.

\section{REFERENCES}

[1] A. A. Sukhorukov, S. Shoji, S. Kawata, and Yu. S. Kivshar, "Self-written waveguides in photosensitive materials," Journal of Nonlinear Optical Physics \& Materials, vol. 11, no. 4, pp. 391-407, 2002.

[2] K. Dorkenoo, O. Crégut, L. Mager, F. Gillot, C. Carre, and A. Fort, "Quasi-solitonic behavior of self-written waveguides created by photopolymerization," Optics Letters, vol. 27, no. 20, pp. 1782-1784, 2002.

[3] V. A. Vdovin, A. L. Lonin, and S. N. Mensov, "Optical waveguide synthesis in photopolymers," Technical Physics, vol. 46, no. 7, pp. 853-857, 2001.

[4] A. L. Lonin and S. N. Mensov, "The optical elongation of lightguides in photopolymerizable compositions," Technical Physics Letters, vol. 28, no. 7, pp. 539-540, 2002.

[5] A. L. Lonin, S. N. Mensov, and Yu. V. Polushtaytsev, "Causes of the filamentary instability of optical-beam self-channeling in photopolymerizable composites," JETP Letters, vol. 79, no. 11, pp. 515-518, 2004.

[6] S. N. Mensov and Yu. V. Polushtaytsev, "Light beams selftrapping process stability in transparent photopolymerizable compositions," in Proceedings of 6th International Conference on Laser and Fiber-Optical Networks Modeling (LFNM'04), pp. 257-259, Kharkov, Ukraine, September 2004.

[7] S. Shoji, S. Kawata, A. A. Sukhorukov, and Yu. S. Kivshar, "Selfwritten waveguides in photopolymerizable resins," Optics Letters, vol. 27, no. 3, pp. 185-187, 2002.

[8] A. L. Lonin and S. N. Mensov, "On the possibility of mutual trapping of counterpropagating light beams in photopolymerizable compositions," Izvestiya Vuzov, Radiophysika, vol. XLVI, no. 2, p. 1, 2002.

[9] S. N. Mensov and Yu. V. Polushtaytsev, "Waveguiding elements optical formation at light beams interaction in transparent photopolymerizable compositions," in Proceedings of the 2nd International Conference on Advanced Optoelectronics and Lasers (CAOL '05), vol. 2, pp. 137-139, Yalta, Ukraine, September 2005.

[10] S. N. Mensov and Yu. V. Polushtaytsev, "Branched waveguiding structures optical formation at light beams crossing in photopolymerizable compositions," Physics of Wave Processes and RadioTechnical Systems, vol. 8, no. 1, p. 51, 2005.
[11] S. N. Mensov and Yu. V. Polushtaytsev, "On the reasons for the mutual interaction of codirectional light beams in a photopolymerizing composition," Optics and Spectroscopy, vol. 100, no. 1, pp. 126-130, 2006.

[12] S. N. Mensov and Yu. V. Polushtaytsev, "Optical formation of waveguiding structures at the interaction of nonintersecting light beams in photopolymerizable compositions," in Proceedings of 8th International Conference on Laser and Fiber-Optical Networks Modeling (LFNM '06), pp. 157-159, Kharkov, Ukraine, June 2006.

[13] M. B. Vinogradova, O. V. Rudenko, and A. P. Sukhorukov, The Theory of Waves, Nauka, Moscow, Russia, 1979.

[14] V. P. Roshchupkin, B. V. Ozerkovsky, and Z. A. Karapetyan, "Structure-kinetic analysis of radical polymerization by modified avrami equation," Vysokomolekulyarnye Soedineniya A, vol. 19, no. 10, p. 2239, 1977.

[15] G. A. Abakumov, O. N. Mamasheva, V. A. Murayev, et al., "Block polymerization method, photopolymerizable composition,” Patent RF RU 2138070, 1999. 

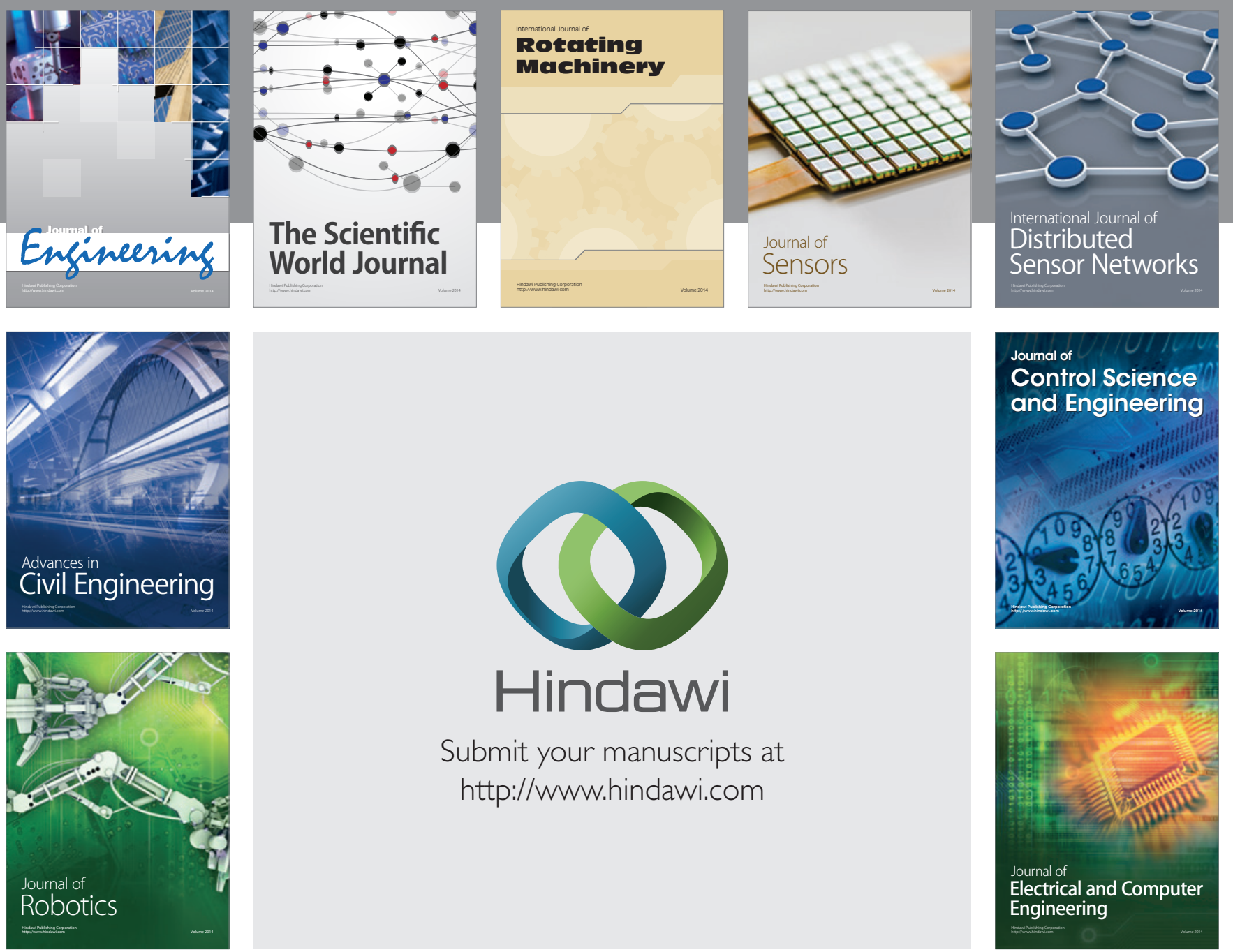

Submit your manuscripts at

http://www.hindawi.com
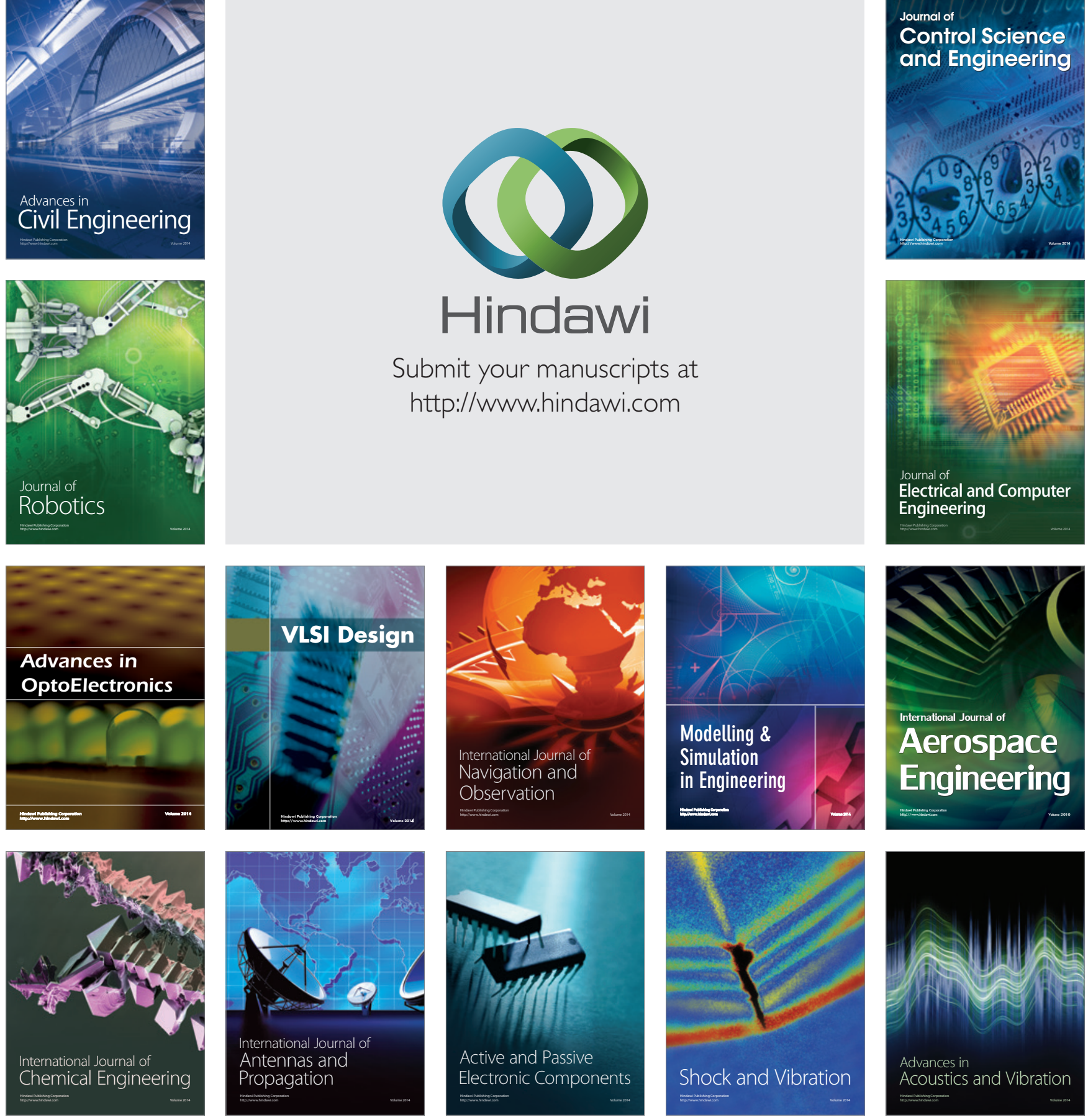